\title{
Design of Inductively Detuned RF Extraction Cavities for the Relativistic Klystron Two Beam Accelerator
}

\author{
E. Henestroza, S.S. Yu, and H. Li \\ Acclerator and Fusion Research Division \\ Lawrence Berkeley Laboratory \\ University of California \\ Berkeley, California 94720
}

\author{
RECEIVED \\ AUG 171995 \\ OSTI
}

April 1995

DISTRIBUTION OF THIS DOCUMENT IS UNLIMITED

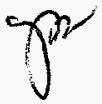

This work was supported by the Director, Office of Energy Research, Office of High Energy Physics, of the U.S. Department of Energy under Contract No. DE-AC03-76SF00098. 


\section{DISCLAIMER}

This report was prepared as an account of work sponsored by an agency of the United States Government. Neither the United States Government nor any agency thereof, nor any of their employees, make any warranty, express or implied, or assumes any legal liability or responsibility for the accuracy, completeness, or usefulness of any information, apparatus, product, or process disclosed, or represents that its use would not infringe privately owned rights. Reference herein to any specific commercial product, process, or service by trade name, trademark, manufacturer, or otherwise does not necessarily constitute or imply its endorsement, recommendation, or favoring by the United States Government or any agency thereof. The views and opinions of authors expressed herein do not necessarily state or reflect those of the United States Government or any agency thereof. 


\section{DISCLAIMER}

Portions of this document may be illegible in electronic image products. Images are produced from the best available original document. 


\title{
DESIGN OF INDUCTIVELY DETUNED RF EXTRACTION CA VITIES FOR THE RELATIVISTIC KLYSTRON TWO BEAM ACCELERATOR*
}

\author{
E. Henestroza, S.S. Yu, and H.Li \\ Lawrence Berkeley Laboratory, \\ University of California \\ Berkeley, California 94720
}

\section{ABSTRACT}

An inductively detuned traveling wave cavity for the Relativistic Klystron Two Beam Accelerator expected to extract high RF power at $11.424 \mathrm{GHz}$ for the $1 \mathrm{TeV}$ Center of Mass Next Linear Collider has been designed. Longitudinal beam dynamics studies led to the following requirements on cavity design:

a) Extraction of $360 \mathrm{MW}$ of RF power with RF component of the current being 1.15 kAmps at 11.424 $\mathrm{GHz}$,

b) Inductively detuned traveling wave cavity with wave phase velocity equal to $4 / 3$ the speed of light,

c) Output cavity with appropriate Qext and eigenfrequency for proper matching.

Furthermore, transverse beam dynamics require low shunt impedances to avoid the beam break-up instability. We describe the design effort to meet these criteria based on frequency-domain and time-domain computations using $2 \mathrm{D}$ - and $3 \mathrm{D}$ - electromagnetic codes.

\section{INTRODUCTION}

An LBL-LLNL team has presented recently a preliminary point design for an $11.424 \mathrm{GHz}$ power source for a $1 \mathrm{TeV}$ Center of Mass Next Linear Collider (NLC) based on the Relativistic-Klystron Two-BeamAccelerator (RK-TBA) concept [1].

The point design requires that the bunched drive beam delivers $360 \mathrm{MW}$ of if power with an if current of $1.15 \mathrm{kA}(600 \mathrm{~A} \mathrm{DC})$ at $11.424 \mathrm{GHz}$ in each of the $150 \mathrm{rf}$ extraction cavities in a 300-m long RK-TBA unit. To achieve this goal, and to maintain longitudinal beam stability over these long distances, the extraction cavities must be inductively detuned; furthermore, in order for a 3-cell disk loaded cavity structure to behave like a traveling wave structure with no reflected waves, the output ports must be properly matched. To maintain low surface fields to avoid breakdown, we consider traveling-wave structures.

*Work supported by the U.S. Department of Energy under Contract DE-AC03-76SF00098.
The required cavity parameters have already been discussed elsewhere in this conference [2]; here we will present electromagnetic calculations to determine the structure of the extraction cavities. We have chosen in this design effort one specific path to meet the general requirements. The procedure adopted is by no means unique, and we anticipate further optimizations and more detailed calculations in the future.

\section{THE EXTRACTION CAVITY DESIGN}

Present designs evolve around traveling-wavestructures with 3 cells of 8 -mm inner radius. The if output is extracted through 2 separate ports in the $3^{\text {rd }}$ cell, with $180 \mathrm{MW}$ each transported through separate waveguides, and fed directly into the two input couplers of the high gradient structure.

The tools we have used to calculate the if properties of the cavity are URMEL and SUPERFISH for 2-D frequency-domain calculations, $\mathrm{ABCI}$ and $\mathrm{TBCI}$ for 2-D time-domain calculations, and MAFIA for 3-D frequency- as well as time-domain calculations. The calculations in frequency-do;nain are obtained for standing-wave solutions. We have followed a procedure described in a paper by Loew at al. [3] to convert these results to traveling-wave properties.

The design procedure is carried out in several steps, starting with the simplest approximations, and adding more realistic features with each successive iteration. At each step we calculate the if properties of the cavity including frequency, the (R/Q), and group velocity for the longitudinal mode, and the synchronous frequency and shunt impedance of the dipole mode; we also calculate the field enhancement factor to determine the surface field for assessing breakdown risks. The field enhancement factor calculated by URMEL is defined as the ratio of the maximum amplitude of the electric field in the whole structure to the average electric field along the axis (including the transit time factor) for standingwave solutions. The enhancernent factor that we are 


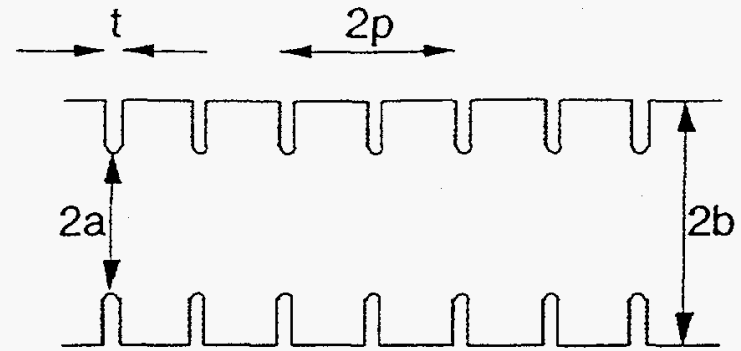

Figure 1: Schematic cross section of the infinitely periodic structure.

interested in is defined for traveling-wave structures without the transit time factor.

\section{INFINTTELY PERIODIC STRUCTURE}

First, we want to determine roughly the geometry of the overall structure. As a starter, we construct a 3-cell disk-loaded traveling-wave structure that is synchronous with the beam $\left(v_{p}=c\right)$. The if structure should give 360 MW with a tightly bunched drive beam with an if current of $1-1.5 \mathrm{kA}$. We choose initially a conventional $2 \pi / 3$ structure with cell length (p) of $8.75 \mathrm{~mm}$. The power extraction formula gives a requirement of $(\mathrm{R} / \mathrm{Q}) / \mathrm{vg}$. URMEL is exercised for an infinitely periodic structure, varying the inner radius $a$ and outer radius $b$ of a disk-loaded structure, and the set of solutions with $v_{p}=c$ is obtained, following the procedure of Thompson, et al. [4]. For each value of the aperture radius satisfying $v_{p}=c$, the cavity parameters $R / Q$ and $v_{g}$ are determined. Figure 1 shows a schematic cross section of the cells.

The inductively detuned structure is next constructed by a variation of the previous step. What we want is a structure whose resonant frequency remains unchanged, but the wavelength is increased by a factor of 1.33 . This will result in a phase velocity of $1.33 \mathrm{c}$, which is the desired detuning angle to maintain longitudinal beam stability. To achieve this, we choose to fix the cell length at $\mathrm{p}=8.75 \mathrm{~mm}$ as before, and reduce the outer radius $b$ for each value of $a$, until the $2 \pi / 3$ field configuration becomes a $\pi / 2$ configuration at the same frequency of $11.424 \mathrm{GHz}$. Each wavelength now extends over 4 cells, instead of the 3 cells. The R/Q and $v_{\mathrm{g}}$ for this new configuration are determined with URMEL. The required geometry is determined by ensuring that the corresponding $R / Q$ and $v_{g}$ provides the right power extraction. The solution is $a=8 \mathrm{~mm}, b=12.5$ $\mathrm{mm}, \mathrm{R} / \mathrm{Q}=13.5 \Omega$ and $\mathrm{vg}_{\mathrm{g}}=0.28 \mathrm{c}$. Slight-refinements from this geometry are obtained by numerically iterating on the power extraction formula to account for the

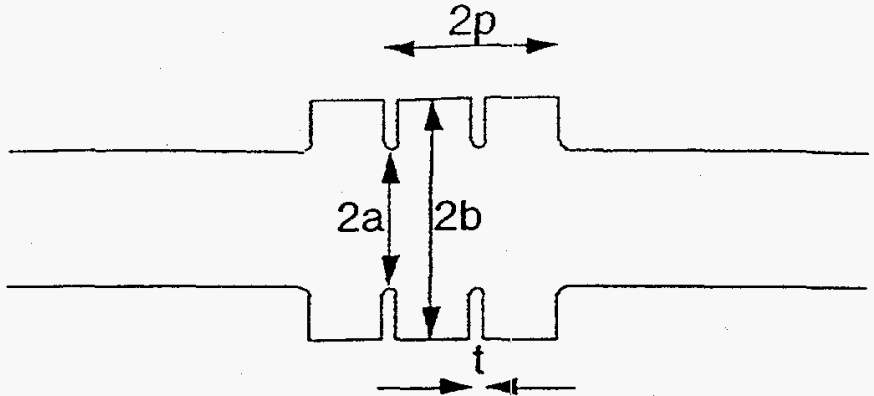

Figure 2: Schematic cross section of the 3-cell cavity with beam pipes.

reduction in power due to inductive detuning. The field enhancement factor is 1.5 .

\section{FINTTE STRUCTIJRE (2-D)}

The finite cell structure is included by modeling the detuned 3-cell structure with finite beam pipes. The effect of the modified geornetry on the field configuration and cavity parameters are then studied. Figure 2. shows a schematic cross section of the 3-cell cavity with beam pipes. As calculated by SUPERFISH a $3 \mathrm{~cm}$ beam pipe on each side of the structure will adequately contain the fringe fields. The cavity fields still retain their $\pi / 2$ structure. Wakefield calculations using $A B C L / B C I$ was performed to find the resonant frequencies and impedances of the structure and to compare the results with the frequency-domain calculations to check for consistency. The resonant frequencies as well as the relative impedance behavior agree with those calculated in frequency-domain.

A very important point to note is that the dipole wake is heavily damped. This is due to the strong coupling of the cavity field to the $\mathrm{TE}_{11}$ mode in the beam pipe. This results in a very low $Q$. To translate the wake calculation to the $Q$ of individual cells in a coupled cavity formulation is sonlewhat subtle, and has not been fully understood yet but initial estimates suggest that the dipole $Q$ for the first and last cavity of our 3-cell structure could be as low as 10 .

\section{FTNTE STRUCTURE (3-D)}

Finally, the 3-D aspects of the output ports are studied using MAFIA. Recalling that the matching condition demands that the frequency and $Q$-value of the last cell be uniquely determined to absorb the reflected waves from the 3-cell structure, MAFIA is exercised by variation of the geometry of the output until the right value of $Q$ and $\omega$ for proper matching are 


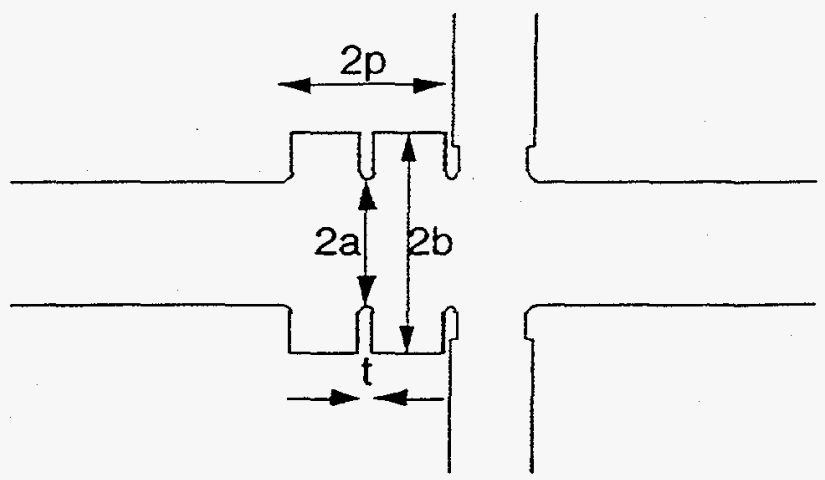

Figure 3: Axial cut of the 3-D cavity with output structures.

obtained. Figure 3 shows an axial cut of the 3-D cavity with output structures.

The output structure consists of two WR90 RWG waveguides attached to the last cell of the cavity. The shorter $\left(0.4^{\prime \prime}\right.$ long) side is oriented along the $z$-axis. The last cell is required to have a $Q_{\text {ext }}<10$ in order to have only a forward traveling wave in the cavity.

The determination of the external $Q$ of the 3-D cavity with output structure is based on the Kroll-Yu method [5]. For a given waveguide iris aperture several calculations (at least four) are performed varying the length of the output waveguide. The phase change along the waveguide, $\psi$, is defined as $2 \pi D / \lambda_{\mathrm{g}}$, where $\lambda_{\mathrm{g}}$ is the waveguide wavelength and $D$ is the waveguide length. The mode of interest (in our case the $\pi / 2$ mode) is identified and the frequency recorded. From the phase change versus frequency relationship the external $Q$ is approximately given by $-(1 / 2) f(d \psi / d f)$. Taking into account the whole structure we calculated $Q_{\text {ext }}=80$ for various waveguide iris apertures. The equivalent $Q$ for the $3^{\text {rd }}$ cavity can be shown, using a method developed in Ref. [1], to be less than 10.

The calculation of the $Q_{\text {ext }}$ of the last cell for $a \pi / 2$ mode configuration is facilitated from the fact that such configuration is obtained naturally by applying neuman and dirichlet boundary conditions at the left and right boundaries of that cell. Using the Kroll-Yu method we calculated a $Q_{e x t}=5$ for a waveguide iris aperture of 1.24 $\mathrm{cm}$, a value of $Q_{\text {ext }}$ that is within that required to meet the matching condition. Figure 4 shows the phase versus frequency curve.

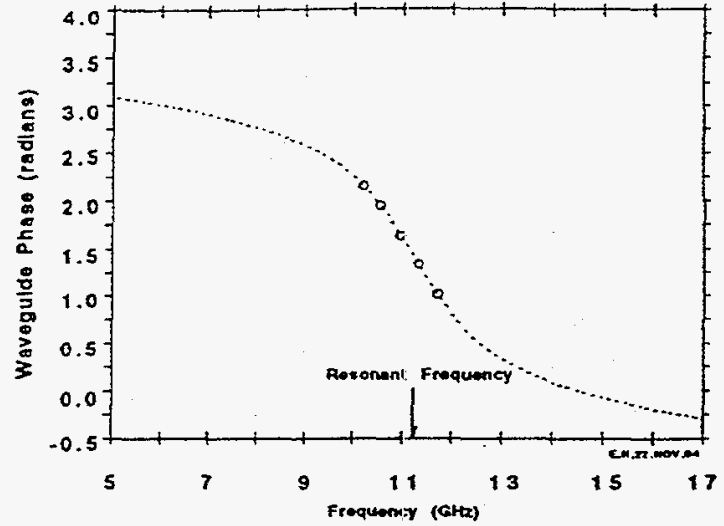

Figure 4: Phase versus frequency curve for last cell.

\section{CONCLUSION}

We do not consider the cavity geometries obtained so far to be final in any serise. There are still large degrees of freedom for design modifications, and further refinements will be conducled to reduce transverse focusing fields and to ensure minimal surface fields over the entire 3-D structure. The calculated Q's are close to the values required to satisfy the matching condition. The induction cavity design need to be optimized to make sure that both the longitudinal as well as transverse impedances are adequately small.

\section{REFERENCES}

[1] S.S. Yu, Deadrick, F., Goffeney, N., Henestroza, E., Houck, T., Li, H., Peters, C., Reginato, L. Sessler, A., Vanecek, D., and Westenskow, G., "Relativistic Klystron Two-Beam Accelerator As A Power Source For A $1 \mathrm{TeV}$ Next Linear Collider Design Report," preliminary draft, LBL, Berkeley, CA, September, 1994.

[2] $\mathrm{H}$. Li, et al., "Beam Dynamics Issues in an Extended Relativistic Klystron, " this conference.

[3] G.A. Loew, R.H. Millier, R.A. Early and K. Bane, "Computer calculations of traveling-wave periodic structure properties", IEEE Trans. Nucl. Science Vol, NS-26. No. 3, June 1979, pp. 3701-3704.

[4] K.A. Thompson, et al, "Design and simulation of accelerating structures for future linear colliders", SLAC-PUB-6032, Nov. 1993.

[5] N.M. Kroll, and D.U.L. Yu, "Computer determination of the external $Q$ and resonant frequency of waveguide loaded structures", Particle Accelerators. 1990. Vol 34, pp. 231-250. 